\title{
Examples of Absolutely Continuous Schrödinger Operators in Magnetic Fields
}

By

\author{
Akira IwATSUKA*
}

\section{§1. Introduction}

In this paper we shall consider the two-dimensional Schrödinger operator $H$ which is the self-adjoint realization in $\mathscr{H}=L^{2}\left(\mathbb{R}^{2}\right)$ of the differential operator

$$
L=\left(\frac{1}{i} \frac{\partial}{\partial x}-a\right)^{2}+\left(\frac{1}{i} \frac{\partial}{\partial y}-b\right)^{2}
$$

where $a$ and $b$ are the operators of multiplication by real-valued $C^{\infty}$ functions $a(x, y)$ and $b(x, y)$, respectively. The spectral property of $H$ depends not directly on the vector potential $(a, b)$ but on the magnetic field

$$
B(x, y)=\frac{\partial b}{\partial x}(x, y)-\frac{\partial a}{\partial y}(x, y),
$$

i.e., all $H$ with $(a, b)$ satisfying (2) with common $B$ are unitarily equivalent to each other (gauge invariance: see, e.g., Leinfelder [5]).

Extensive studies have been made in the case where $B$ is asymptotically constant, that is,

$$
B(x, y) \longrightarrow B_{0} \text { as } \sqrt{x^{2}+y^{2}} \longrightarrow \infty,
$$

where $B_{0}$ is some constant. In the case where $B_{0}=0$, the essential spectrum $\sigma_{\text {ess }}(H) \equiv\{\lambda \in \mathbb{R} \mid \operatorname{dim} \operatorname{Ran}(E((\lambda-\varepsilon, \lambda+\varepsilon)))=\infty$ for all $\varepsilon>0\}$ of $H$ is $[0, \infty)$ (see [5]), where $E$ denotes the spectral measure associated with $H$. Moreover, if $B$ is short-range (i.e., $B=O\left({\sqrt{x^{2}+y^{2}}}^{-1-\delta}\right)$ for some $\left.\delta>0\right), H$ is absolutely continuous, i.e., the subspace of absolute continuity $\mathscr{H}_{a c} \equiv\{u \in \mathscr{H} \mid \| E((-\infty$, 2]) $u \|^{2}$ is absolutely continuous $\}$ for $H$ fills up the whole space $\mathscr{H}$ (see Ikebe and Saito [2]). In the long-range case, there is an example of $H$ with pure point

Communicated by S. Matsuura, February 15, 1984.

* Department of Mathematics, Kyoto University, Kyoto 606, Japan. 
spectrum in the sense that $\mathscr{H}$ is spanned by the eigenvectors of $H$ (see Miller and Simon [7]). On the other hand, in the case where $B_{0} \neq 0$, the operator $H$ shows a completely different spectral behavior. Namely, we have shown in [3] that

$$
\sigma_{\text {ess }}(H)=\left\{(2 k+1)\left|B_{0}\right| \mid k \text { is an integer } \geqq 0\right\},
$$

which implies that $H$ has pure point spectrum (see also Avron, Herbst and Simon [1]). However, as far as we know, the case where $B$ is not asymptotically constant has not been studied very well. We shall consider the case where $B$ satisfies the following property:

(B) $B(x, y)$ depends only on $x$ (i.e., $B(x, y)=B(x)), B(x)$ is a $C^{\infty}$ function such that there exist constants $M_{ \pm}$satisfying $0<M_{-} \leqq B(x) \leqq M_{+}<\infty$ for all $x$.

Let $\dot{H}$ be the operator in $\mathscr{H}=L^{2}\left(\boldsymbol{R}^{2}\right)$ defined on the space $C_{0}^{\infty}\left(\boldsymbol{R}^{2}\right)$ of all $C^{\infty}$ functions with compact support by

$$
\dot{H} u=L u \quad \text { for } \quad u \in C_{0}^{\infty}\left(\boldsymbol{R}^{2}\right) .
$$

Let $H$ be the closure of $\dot{H}$. Then $\dot{H}$ is essentially self-adjoint (see Leinfelder and Simader [6]) and hence $H$ is the unique self-adjoint extention of $\dot{H}$. Therefore, the adjoint operator $\dot{H}^{*}$ of $\dot{H}$ equals to $H$ and, by examining $\dot{H}^{*}$, one can obtain

$$
\left\{\begin{array}{l}
D(H)=\{f \in \mathscr{H} \mid L f \in \mathscr{H}\}, \\
H f=L f \quad \text { for } \quad f \in D(H),
\end{array}\right.
$$

where and in the sequel differentiation is understood in the distribution sense. The aim of the present paper is to show that $H$ is absolutely continuous if either of the following (B1) or (B2) holds:

(B1) In addition to (B), $\limsup _{x \rightarrow-\infty} B(x)<\liminf _{x \rightarrow+\infty} B(x)$ or $\limsup _{x \rightarrow+\infty} B(x)<$ $\lim _{x \rightarrow-\infty} B(x)$.

(B2) In addition to (B), $B(x)=B_{0}$ for some constant $B_{0}$ if $|x|$ is sufficiently large, and there exists a point $\bar{x}$ such that $B^{\prime}(x) \leqq 0$ for $x \leqq \bar{x}$ and $B^{\prime}(x) \geqq 0$ for $x \geqq \bar{x}$ (or $B^{\prime}(x) \geqq 0$ for $x \leqq \bar{x}$ and $B^{\prime}(x) \leqq 0$ for $x \geqq \bar{x}$ ) and $B^{\prime}(x)$ is not identically 0 .

Theorem. Suppose that either (B1) or (B2) holds. Then $H$ is absolutely continuous. 


\section{§2. Reduction to One-Dimensional Hamiltonians}

Under the assumption (B), we can take the vector potential $(a, b)$ of the form

$$
a=0, b=b(x)=\int_{0}^{x} B(t) d t
$$

in view of (2). Therefore, since we shall assume (B) throughout the paper, we shall henceforth consider the differential operator

$$
L=-\frac{\partial^{2}}{\partial x^{2}}+\left(\frac{1}{i} \frac{\partial}{\partial y}-b(x)\right)^{2} .
$$

Let $H_{1}$ be the operator in $\mathscr{H}$ defined on the Schwartz space $\mathscr{S}\left(\mathbb{R}^{2}\right)$ of rapidly decreasing $C^{\infty}$ functions by

$$
H_{1} u=L u \quad \text { for } \quad u \in \mathscr{S}\left(\mathbb{R}^{2}\right)
$$

(note that $L u \in \mathscr{H}$ for $u \in \mathscr{S}\left(\boldsymbol{R}^{2}\right)$ by the estimate $|b(x)| \leqq M_{+}|x|$ obtainable from (4) and (B)). Then $\dot{H} \subset H_{1}$ and $H_{1} \subset H$ by (3). Thus $H_{1}$ is essentially selfadjoint since $\dot{H}$ is so. Let $\widetilde{L}$ be the differential operator

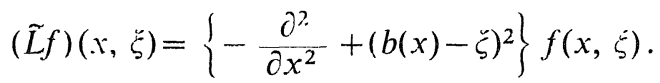

Then it is clear that $\tilde{L} \mathscr{F} u=\mathscr{F} L u$ for $u \in \mathscr{S}\left(\mathbb{R}^{2}\right)$ where $\mathscr{F}$ is the partial Fourier transformation

$$
\mathscr{F} u(x, \xi)=(2 \pi)^{-1 / 2} \int e^{-i y \xi} u(x, y) d y .
$$

Let $\tilde{H}_{1}$ be the operator in $\tilde{\mathscr{H}}=L^{2}\left(\mathbb{R}_{x} \times \mathbb{R}_{\xi}\right)$ defined by $\tilde{H}_{1} f=\tilde{L} f$ for $f \in D\left(\tilde{H}_{1}\right) \equiv$ $\mathscr{S}\left(\boldsymbol{R}_{x} \times \boldsymbol{R}_{\xi}\right)$, and let $\tilde{H}$ be the closure of $\tilde{H}_{1}$. Then $\tilde{H}_{1}=\mathscr{F} H_{1} \mathscr{F}^{-1}$ and hence, since $\mathscr{F}$ is unitary and $H_{1}$ is essentially self-adjoint, $\widetilde{H}_{1}$ is essentially self-adjoint. Therefore, $\widetilde{H}$ is self-adjoint and $\widetilde{H}=\mathscr{F} H_{F}^{-1}$. Thus we have

Lemma 2.1. Assume that (B) holds. Let $\tilde{H}$ be the self-adjoint operator clefined above. Then $H$ is unitarily equivalent to $\tilde{H}$.

Lemma 2.2. $C_{0}^{\infty}\left(\boldsymbol{R}_{x} \times \boldsymbol{R}_{\xi}\right)$ is a core for $\tilde{H}$ and

$$
\left\{\begin{array}{l}
D(\tilde{H})=\{f \in \tilde{\mathscr{H}} \mid \tilde{L} f \in \tilde{\mathscr{H}}\} \\
\tilde{H} f=\tilde{L} f \quad \text { for } \quad f \in D(\tilde{H}) .
\end{array}\right.
$$


Proof. Let $\zeta \in C_{0}^{\infty}\left(\boldsymbol{R}_{x} \times \boldsymbol{R}_{\xi}\right)$ such that $\zeta(x, \xi)=1$ for $\sqrt{x^{2}+\xi^{2}} \leqq 1$ and $0 \leqq \zeta \leqq 1$. Then it is not difficult to verify that, for $f \in \mathscr{S}\left(\boldsymbol{R}_{x} \times \boldsymbol{R}_{\xi}\right), \zeta_{n} f \rightarrow f$, $\tilde{L}\left(\zeta_{n} f\right) \rightarrow \tilde{L} f$ strongly in $\widetilde{\mathscr{H}}$ as $n \rightarrow \infty$, where $\zeta_{n}$ is the operator of multiplication by $\zeta(x / n, \xi / n)$ for $n=1,2, \ldots$ Hence, since $\mathscr{S}\left(\boldsymbol{R}_{x} \times \boldsymbol{R}_{\xi}\right)$ is a core for $\tilde{H}, C_{0}^{\infty}\left(\boldsymbol{R}_{x}\right.$ $\left.\times \boldsymbol{R}_{\xi}\right)$ is a core for $\tilde{H}$. Moreover, it follows that $\tilde{H}$ coincides with the adjoint operator of $\left.\widetilde{H}\right|_{C_{0}^{\infty}\left(\boldsymbol{R}_{x} \times \boldsymbol{R}_{\xi}\right)}(\mid$ denotes the restriction), from which (7) follows.

$\tilde{L}$ can be written as

$$
\tilde{L} f(x, \xi)=(\tilde{L}(\xi) f(\cdot, \xi))(x),
$$

where $\tilde{L}(\xi)$ is a second-order ordinary differential operator

$$
\tilde{L}(\xi)=-\frac{d^{2}}{d x^{2}}+(b(x)-\xi)^{2} .
$$

Let $\dot{\tilde{H}}(\xi)$ be the operator in $L^{2}\left(\boldsymbol{R}_{x}\right)$ defined by $\dot{\tilde{H}}(\xi) \phi=\widetilde{L}(\xi) \phi$ for $\phi \in D(\dot{\tilde{H}}(\xi))$ $\equiv C_{0}^{\infty}\left(\boldsymbol{R}_{x}\right)$, and let $\widetilde{H}(\xi)$ be the closure of $\dot{\tilde{H}}(\xi)$. Then, since $\widetilde{L}(\xi)$ is in the limit point case at $\pm \infty$ (see, e.g., [8], Appendix to X.1), $\dot{\widetilde{H}}(\xi)$ is essentially self-adjoint and hence $\tilde{H}(\xi)$ is self-adjoint.

Lemma 2.3. Assume that $(B)$ holds. Let $\xi$ be a real number and let $\tilde{H}(\xi)$ be the self-adjoint operator defined above. Then there exists a complete

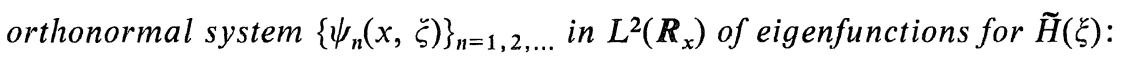

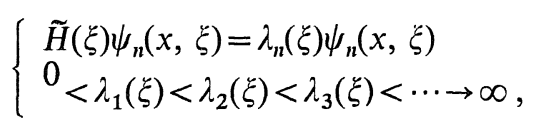

so that, for $n=1,2, \ldots$,

(i) each $\lambda_{n}(\xi)$ is non-degenerate,

(ii) $(2 n-1) M_{-} \leqq \lambda_{n}(\xi) \leqq(2 n-1) M_{+}$,

(iii) $\lambda_{n}(\xi)$ depends analytically on $\xi$,

(iv) $\psi_{n}(\cdot, \xi) \in D(\tilde{H}(0))$ and depends analytically on $\xi$ with respect to the graph norm $\|u\| \equiv\left(\|u\|^{2}+\|\tilde{H}(0) u\|^{2}\right)^{1 / 2}$,

(v) $\psi_{n}(x, \xi)$ is a real-valued continuous function of $x$ and $\xi$, and, moreover, $\psi_{n}(x, \xi)$ is infinitely differentiable in $x$ for each $\xi$ and is analytic in $\xi$ for each $x$.

Proof. First part ((i) and (ii)): Since $d b(x) / d x=B(x) \geqq M_{-}>0$ by (B) and (4), $b(x)$ is a strictly increasing function of $x$ such that $\lim _{x \rightarrow \pm \infty} b(x)= \pm \infty$. Thus the equation $b(x)=\xi$ has a unique solution for each $\xi$, which we shall denote by $x_{\xi}$ (i.e., $x_{\xi} \equiv b^{-1}(\xi)$ ). Then we have 


$$
b(x)-\xi=\int_{x_{\xi}}^{x} B(t) d t
$$

Hence we have from the assumption (B)

$$
\begin{aligned}
& M_{-}\left(x-x_{\xi}\right) \leqq b(x)-\xi \leqq M_{+}\left(x-x_{\xi}\right) \quad \text { for } \quad x \geqq x_{\xi} \text {, } \\
& M_{-}\left(x-x_{\xi}\right) \geqq b(x)-\xi \geqq M_{+}\left(x-x_{\xi}\right) \quad \text { for } \quad x \leqq x_{\xi} \text {, }
\end{aligned}
$$

from which we obtain the following inequality for all $x$ :

$$
M_{-}^{2}\left(x-x_{\xi}\right)^{2} \leqq(b(x)-\xi)^{2} \leqq M_{+}^{2}\left(x-x_{\xi}\right)^{2} .
$$

Since $(b(x)-\xi)^{2}$ is smooth and tends to $\infty$ as $|x| \rightarrow \infty$ by (12), $\tilde{H}(\xi)$ has compact resolvent and a complete set of eigenfunctions $\left\{\psi_{n}(\cdot, \xi)\right\}_{n=1,2, \ldots}$ with eigenvalues $\left\{\lambda_{n}(\xi)\right\}_{n=1,2, \ldots}$ such that $\lambda_{1}(\xi) \leqq \lambda_{2}(\xi) \leqq \lambda_{3}(\xi) \leqq \cdots \rightarrow \infty$ (see, e.g., [9], Theorems XIII. 64 and XIII. 67). The proof of the non-degeneracy of $\lambda_{n}(\xi)$ needs a proposition concerning the eigenfunctions of second order differential equations, which we shall prove in the next section (Proposition 3.1). By Proposition 3.1 with $q(x)=(b(x)-\xi)^{2}-\lambda_{n}(\xi)$ and $l=\left[x_{0}, \infty\right)$ where $x_{0}=x_{\xi}+\sqrt{\lambda_{n}(\xi)} / M_{-}(q(x)$ $\geqq 0$ on $I$ by (12)), any square integrable real-valued solution of the differential equation

$$
\widetilde{L}(\zeta) u(x)=\lambda_{n}(\zeta) u(x)
$$

satisfies the inequality

$$
u^{\prime}(x) u(x)<0 \quad\left({ }^{\prime} \text { denotes } \frac{d}{d x}\right)
$$

for $x \geqq x_{0}$. On the other hand, since $\tilde{L}(\xi)$ is of second order, the multiplicity of $\lambda_{n}(\xi)$ is 1 or 2 . If the multiplicity is 2 , all the solutions of (13) would belong to $L^{2}\left(\mathbb{R}_{x}\right)$. But this is impossible because we can solve the equation (13) with given initial value of $\left(u, u^{\prime}\right)$, say, $(1,1)$ at the point $x_{0}$, which contradicts (14). This implies that the multiplicity of $\lambda_{n}(\xi)$ is 1 , i.e., (i) holds and hence $\lambda_{1}(\xi)<\lambda_{2}(\xi)<$ $\lambda_{3}(\xi)<\cdots$.

Next, from (12) and a comparison theorem based on the min-max principle (see, e.g.. [9], p. 270, Lemma), we have (ii) since the $n$-th eigenvalue of the harmonic oscillator Hamiltonian $-\frac{d^{2}}{d x^{2}}+M_{ \pm}^{2}\left(x-x_{\xi}\right)^{2}$ is $(2 n-1) M_{ \pm}$.

For the remainder of the proof of Lemma 2.3, we need a lemma (Lemma 2.4 below). Let $\mathscr{D}$ denote the space $D(\tilde{H}(0))$ equipped with the graph norm \|\|$\cdot \|, P_{n}(\xi)$ the projection onto the $n$-th eigenspace of $\tilde{H}(\xi)$ and $\mathbb{B}(X, Y)$ the space 
of bounded operators from $X$ to $Y$ for Banach spaces $X$ and $Y$.

Lemma 2.4. (a) The operator $b$ is relatively bounded with relative bound 0 with respect to $\tilde{H}(0)$.

(b) $D(\tilde{H}(\xi))$ equipped with its graph norm $\|u\|_{D(\tilde{H}(\xi))} \equiv\left(\|u\|^{2}+\|\tilde{H}(\xi) u\|^{2}\right)^{1 / 2}$

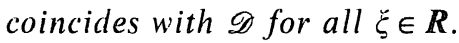

(c) $\mathscr{D}$ is a subspace of $C_{0}(\boldsymbol{R}) \equiv\{f \mid f$ is a continuous function on $\boldsymbol{R},|f(x)| \rightarrow 0$ as $|x| \rightarrow \infty$, and the inclusion map: $\mathscr{D} \rightarrow C_{0}(\boldsymbol{R})$ belongs to $\boldsymbol{B}\left(\mathscr{D}, C_{0}(\boldsymbol{R})\right)$, where $C_{0}(\boldsymbol{R})$ is equipped with the norm $|f|_{\infty} \equiv \sup _{x \in \boldsymbol{R}}|f(x)|$.

(d) $P_{n}(\xi)$ is a $\boldsymbol{B}(\mathscr{H}, \mathscr{D})$-valued analytic function of $\xi \in \boldsymbol{R}$.

Proof. (a) We have in view of (9)

$$
\|b u\|^{2}=\left(b^{2} u, u\right) \leqq(\tilde{L}(0) u, u) \leqq \varepsilon\|\tilde{L}(0) u\|^{2}+\frac{1}{2 \varepsilon}\|u\|^{2},
$$

for all $u \in C_{0}^{\infty}(\boldsymbol{R})$ and for all $\varepsilon>0$, where ( , ) denotes the inner product of $L^{2}\left(\boldsymbol{R}_{x}\right)$. This inequality implies that the operator $b$ is relatively bounded with relative bound 0 with respect to $\dot{\tilde{H}}(0)$ and hence with respect to $\tilde{H}(0)=$ the closure of $\dot{\tilde{H}}(0)$.

(b) We have by (9)

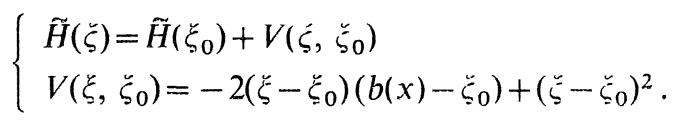

It is not difficult to verify, by using (a) and (15) with $\breve{\zeta}_{0}=0$, that the norm $\|\cdot \mid\|$ and the norm $\|\cdot\|_{D(\tilde{H}(\xi))}$ are equivalent on $C_{0}^{\infty}(\boldsymbol{R})$. Therefore, since $D(\tilde{H}(\xi))$ coincides with the completion of $C_{0}^{\infty}(R)$ with respect to the norm $\|\cdot\|_{D(\tilde{H}(\xi))}$ by the definition of closure of an operator, we have (b).

(c) Since we have a Sobolev inequality

$$
|f|_{x} \leqq C\left(\|f\|+\left\|\frac{d}{d x} f\right\|\right)
$$

for $f \in C_{0}^{\infty}(\boldsymbol{R})$ where $C$ is a constant and we have in view of (9)

$$
\left\|\frac{d}{d x} f\right\|^{2}=-\left(\frac{d^{2} f}{d x^{2}}, f\right) \leqq(\tilde{L}(0) f, f) \leqq-\frac{1}{2}\left(\|f\|^{2}+\|\tilde{L}(0) f\|^{2}\right)
$$

for $f \in C_{0}^{\infty}(\boldsymbol{R})$, we obtain

$$
|f|_{\infty} \leqq C|| f||
$$

for $f \in C_{0}^{\infty}(\boldsymbol{R})$. From this inequality it follows that the identity map on $C_{0}^{\infty}(\boldsymbol{R})$ can be extended to a continuous one to one map from the completion $\mathscr{D}$ of 
$C_{0}^{\infty}(\boldsymbol{R})$ with respect to the norm $\|\cdot\|$ into the completion $X$ of $C_{0}^{\infty}(\mathbb{R})$ with respect to the norm $|\cdot|_{\alpha_{\alpha}}$. Since it is not difficult to check that $X$ is $C_{0}(\mathbb{R})$, we obtain (c).

(d) Fix $\xi_{0}$. Then it is clear by (b) that $\left(\tilde{H}\left(\xi_{0}\right)-\lambda\right)^{-1}$ is a $\mathbb{B}(\mathscr{H}, \mathscr{D})$-valued continuous (analytic) function of $\lambda \notin \sigma\left(\tilde{H}\left(\xi_{0}\right)\right)$. Let $\Gamma$ be a circle $\{\lambda|| \lambda-$ $\lambda_{n}\left(\xi_{0}\right) \mid=\varepsilon$, in the complex plane $C$ for some integer $n$ with sufficiently small $\varepsilon>0$ such that $\sigma\left(\tilde{H}\left(\xi_{0}\right)\right) \cap\left\{\lambda \in C|| \lambda-\lambda_{n}\left(\xi_{0}\right) \mid \leqq 2 \varepsilon\right\}=\left\{\lambda_{n}\left(\xi_{0}\right)\right\}$. Then, there exists a constant $K>0$ such that $\left\|\left(\tilde{H}\left(\xi_{0}\right)-\lambda\right)^{-1}\right\|_{\boldsymbol{B}(\mathscr{C}, \mathscr{Q})} \leqq K$ for $\lambda \in \Gamma$. Let $\Delta=\{\dot{\xi} \in \mathbb{C} \mid$ $\left.\left|\xi-\xi_{0}\right|<\delta\right\}$ with sufficiently small $\delta>0$ such that $\left\|V\left(\xi, \xi_{0}\right)\right\|_{\boldsymbol{B}(\mathscr{\rho}, \mathscr{L})} \leqq \frac{1}{2 K}$ for $\xi \in \Delta$, where $V\left(\breve{\zeta}, \breve{\zeta}_{0}\right)$ is as in (15). Then the Neumann series

$$
\left(1+V\left(\xi, \dot{\zeta}_{0}\right)\left(\tilde{H}\left(\xi_{0}\right)-\lambda\right)^{-1}\right)^{-1}=\sum_{j=0}^{\infty}\left\{V\left(\check{\zeta}, \xi_{0}\right)\left(\tilde{H}\left(\xi_{0}\right)-\lambda\right)^{-1}\right\}^{j}
$$

converges in $\boldsymbol{B}(\mathscr{H}, \mathscr{H})$ uniformly for $(\lambda, \xi) \in \Gamma \times \Delta$, and hence becomes a $\boldsymbol{B}(\mathscr{H}$, $\mathscr{H})$-valued continuous function of $(\lambda, \xi) \in \Gamma \times \Delta$ which is analytic in $\xi \in \Delta$. Hence, we have by (15)

$$
(\tilde{H}(\zeta)-\lambda)^{-1}=\left(\tilde{H}\left(\check{\zeta}_{0}\right)-\lambda\right)^{-1}\left(1+V\left(\zeta, \zeta_{0}\right)\left(\tilde{H}\left(\zeta_{0}\right)-\lambda\right)^{-1}\right)^{-1},
$$

for $(\lambda, \breve{\zeta}) \in \Gamma \times \Delta$, which is a $\mathbb{B}(\mathscr{H}, \mathscr{D})$-valued continuous function of $(\lambda, \breve{\zeta}) \in$ $\Gamma \times \Delta$, analytic in $\xi$. Consequently $P_{n}(\xi)$ is a $B(\mathscr{H}, \mathscr{D})$-valued analytic function of $\check{\zeta} \in \Delta$, since we have ([9], Theorem XII. 5) for $\check{\zeta} \in \Delta$

$$
P_{n}(\dot{\zeta})=\frac{-1}{2 \pi i} \int_{1}(\tilde{H}(\xi)-\lambda)^{-1} d \lambda
$$

Thus, since $\zeta_{0}$ was arbitrary, we obtain $(\mathrm{d})$.

Proof of Lemma 2.3. Second part ((iii), (iv) and (v)): $D(\widetilde{H}(\xi))=D(\widetilde{H}(0))$ by (b) of Lemma 2.4 and $\tilde{H}(\xi) u$ is analytic in $\xi$ for all $u \in D(\tilde{H}(0))$. Hence, $\tilde{H}(\xi)$ is an analytic family of type (A) (see [4], p. 345). Thus $\lambda_{n}(\zeta)$ depends analytically on $\dot{\zeta}([4]$, p. 370 , Theorem 1.8$)$ since $\lambda_{n}(\zeta)$ is non-degenerate by (i). This proves (iii).

For (iv) and (v), we first show the existence of an $\mathscr{H}$-valued analytic function $\psi_{n}(\cdot, \xi)$ of $\xi \in \mathbb{R}$ such that $\psi_{n}(\cdot, \xi)$ is a real and normalized eigenfunction of $\tilde{H}(\zeta)$ with eigenvalue $\lambda_{n}(\xi)$. Since $\widetilde{H}(\xi)$ is real (i.e., commutes with the complex conjugation $C: C u=\bar{u})$, it follows from (16) that $P_{n}(\xi)$ is real. Morcover, $P_{n}(\xi)$ is a projection and a $\mathbb{B}(\mathscr{H}, \mathscr{H})$-valued analytic function of $\xi$ by (d) of Lemma 2.4 since the inclusion map: $\mathscr{D} \rightarrow \mathscr{H}$ is continuous. Thus we can make usc of Theorem XII.12 of [9] which guarantces the existence of an analytic 
family $U_{n}(\xi)$ of unitary operators such that

$$
U_{n}(\xi) P_{n}(0)=P_{n}(\xi) U_{n}(\xi)
$$

By examining the construction of $U_{n}(\xi)$ (see the proof of Theorem XII. 12 in [9]), it is not difficult to verify that, if $C P_{n}(\xi) C=P_{n}(\xi)$ for all real $\xi, C U_{n}(\xi) C=$ $U_{n}(\xi)$ for all real $\xi$. Therefore, if we let $\psi_{n}(\cdot, 0)$ be a real and normalized element of $\operatorname{Ran}\left(P_{n}(0)\right)$ and let $\psi_{n}(\cdot, \xi) \equiv U_{n}(\xi) \psi_{n}(\cdot, 0)$, then it is not difficult to check that $\psi_{n}(\cdot, \xi)$ is real, normalized, analytic in $\xi$ and an eigenfunction of $\widetilde{H}(\xi)$ with eigenvalue $\lambda_{n}(\xi)$ since $\psi_{n}(\cdot, \xi)$ belongs to $\operatorname{Ran}\left(P_{n}(\xi)\right)$ by (17). Thus (iv) holds by (d) of Lemma 2.4 since $\psi_{n}(\cdot, \xi)=P_{n}(\xi) \psi_{n}(\cdot, \xi)$.

Finally (v) can be shown as follows: $\psi_{n}(\cdot, \xi)$ is real-valued as it has been shown in the above. By (iv) and (c) of Lemma $2.4 \psi_{n}(\cdot, \xi)$ is a $C_{0}(\mathbb{R})$-valued analytic function of $\xi$, from which it follows that $\psi_{n}(x, \xi)$ is continuous in $x$ and $\xi$, and analytic in $\xi$ for each $x$. The smoothness of $\psi_{n}(x, \xi)$ in $x$ for each $\xi$ follows from (10) and the smoothness of $b(x)$.

Lemma 2.5. Assume that (B) holds. Let $\widetilde{\mathscr{H}}_{n}$ be the subspace of $\widetilde{\mathscr{P}}=$ $L^{2}\left(\boldsymbol{R}_{x} \times \boldsymbol{R}_{\xi}\right)$ defined by

$$
\widetilde{\mathscr{H}}_{n}=\left\{\psi_{n}(x, \xi) f(\xi) \mid f(\xi) \in L^{2}\left(\boldsymbol{R}_{\xi}\right)\right\} \quad(n=1,2, \ldots)
$$

where $\psi_{n}(x, \xi)$ is as in Lemma 2.3. Then we have:

(i) $\widetilde{\mathscr{H}}=\bigoplus_{n} \widetilde{\mathscr{H}}_{n}$ (orthogonal sum).

(ii) $\tilde{H}$ is reduced by $\widetilde{\mathscr{H}}_{n}$.

(iii) $\left.\tilde{H}\right|_{\tilde{\mathscr{H}}_{n}}$ (the restriction of $\tilde{H}$ to $\widetilde{\mathscr{H}}_{n}$ ) is unitarily equivalent to the operator of multiplication by $\lambda_{n}(\xi)$ on $L^{2}\left(\boldsymbol{R}_{\xi}\right)$.

Proof. Since $\left\{\psi_{n}(\cdot, \xi)\right\}$ is a complete orthonormal system in $L^{2}\left(\boldsymbol{R}_{x}\right)$ by Lemma 2.3, (i) holds.

It follows from (8) and (10) that

$$
\tilde{L}\left\{\psi_{n}(x, \xi) f(\xi)\right\}=\lambda_{n}(\xi) \psi_{n}(x, \xi) f(\xi) .
$$

The right-hand side of (18) belongs to $L^{2}\left(\boldsymbol{R}_{x} \times \boldsymbol{R}_{\xi}\right)$ since $\lambda_{n}(\xi)$ is bounded in $\xi$ by Lemma 2.3 (ii). Hence $\tilde{f} \in D(\tilde{H})$ and $\tilde{H} \tilde{f}=\lambda_{n}(\xi) \tilde{f}$ for $\tilde{f} \in \widetilde{\mathscr{H}}_{n}$ by (7) and (18). Thus we have that $\widetilde{\mathscr{H}}_{n} \subset D(\widetilde{H})$ and $\widetilde{H}\left(\widetilde{\mathscr{H}}_{n}\right) \subset \widetilde{\mathscr{H}}_{n}$. This implies that $\tilde{H}$ is reduced by $\widetilde{\mathscr{H}}_{n}$. Hence we obtain (ii).

If we define $T_{n}: L^{2}\left(\boldsymbol{R}_{\xi}\right) \rightarrow L^{2}\left(\boldsymbol{R}_{x} \times \boldsymbol{R}_{\xi}\right)$ such that $T_{n} f(x, \xi)=\psi_{n}(x, \xi) f(\xi)$, then $\tilde{H} T_{n} f=T_{n}\left\{\lambda_{n} f\right\}$ by (18) where $\lambda_{n}$ denotes the operator of multiplication by $\lambda_{n}(\xi)$. Thus we have (iii) since $T_{n}$ is an isometry with the range $\widetilde{\mathscr{H}}_{n}$ and $\widetilde{H} G_{n}=$ 
$\tilde{H} T_{n} T_{n}^{*}=T_{n} \lambda_{n} T_{n}^{*}$, where $G_{n}$ denotes the orthogonal projection onto $\widetilde{\mathscr{H}}_{n}$. This completes the proof of the lemma.

Lemma 2.6. Assume that (B) holds. Then $H$ is absolutely continuous if no $\lambda_{n}(\xi)$ is constant, where $\lambda_{n}(\xi)$ is as in Lemma 2.3 .

Proof. By Lemma 2.1 and Lemma 2.5, it suffices to show that, for each $n$, $\lambda_{n}$ is absolutely continuous if $\lambda_{n}(\xi)$ is not constant. Since $\lambda_{n}(\xi)$ is analytic and non-constant, $A \equiv\left\{\xi \mid \frac{d}{d \xi} \lambda_{n}(\xi)=0\right\}$ is discrete and closed. Hence, if we let $\left\{I_{j}\right\}_{j \in J}$ be the connected components of $\mathbb{R} \backslash A$, then $I_{j}$ are open intervals, $J$ is at most countable, and

$$
L^{2}(\boldsymbol{R})=\underset{j \in J}{\oplus} L^{2}\left(I_{j}\right) \quad \text { (orthogonal sum). }
$$

Hence it suffices to show that the operator $\Lambda_{j}$ of multiplication by $\lambda_{n}(\xi)$ on $L^{2}\left(I_{j}\right)$ is absolutely continuous for each $j \in J$. On each interval $I_{j}, \lambda_{n}(\xi)$ is strictly monotone, either increasing or decreasing. Consider the case where it is increasing. Let $\alpha$ denote the inverse function of the restriction of $\lambda_{n}(\xi)$ to $I_{j}$. Then $\alpha$ is a strictly increasing smooth function on $\lambda_{n}\left(I_{j}\right)$. Let $E_{\mu}$ be the spectral measure associated with $\Lambda_{j}$. Then we have

$$
\begin{aligned}
\left\|E_{\mu} u\right\|^{2} & =\int_{\left\{\xi \in I_{J} \mid \lambda_{n}(\xi)<\mu\right\}}|u(\zeta)|^{2} d \xi \\
& =\int_{a}^{\alpha(\mu)}|u(\zeta)|^{2} d \zeta \quad\left(a=\inf I_{j}\right) \\
& =f_{u}(\alpha(\mu))
\end{aligned}
$$

for $u \in L^{2}\left(I_{j}\right)$, where $f_{u}(x)=\int_{a}^{x}|u(\xi)|^{2} d \xi$ and where we extend $\alpha$ to all the real line so that $\alpha(\mu)=\sup I_{j}$ if $\mu \geqq \sup \lambda_{n}\left(I_{j}\right)$ and $\alpha(\mu)=a$ if $\mu \leqq \inf \lambda_{n}\left(I_{j}\right)$. Therefore one can verify without difficulty that the absolute continuity of $\alpha$ (which follows from the smoothness) and the monotonicity of $\alpha$ together with the absolute continuity of $f_{u}$ imply the absolute continuity of $\left\|E_{\mu} u\right\|^{2}$. Thus $\Lambda_{j}$ is an absolutely continuous operator.

\section{§3. Properties of $\psi_{n}(x, \xi)$ and $\lambda_{n}(\xi)$}

Throughout this section, we suppose (B) alone, use the notations in Lemma 2.3 and let $n$ be fixed. In addition, we put

$$
Q_{n, \xi}(x) \equiv(b(x)-\xi)^{2}-\lambda_{n}(\xi) \text {. }
$$


Then, by (10), we have

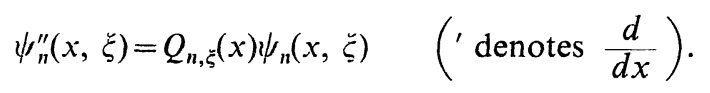

In this section we shall invesigate some detailed properties of the eigenfunctions

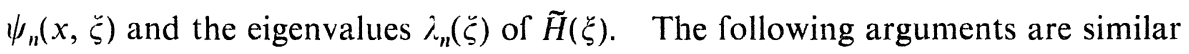
to those in [10] (p. 110 and p. 165 fr.).

Proposition 3.1. Let $I=\left[x_{0}, \infty\right)\left(\left(-\infty, x_{0}\right]\right)$. Let $u$ be a real-valued $C^{2}$ function and $q$ a non-negative continuous function on $I$. Suppose that u satisfies

$$
u^{\prime \prime}(x)=q(x) u(x),
$$

$u \in L^{2}(I)$ and $u \neq \equiv 0$. Then $u(x) u^{\prime}(x)<0$ for $x \in I\left(u(x) u^{\prime}(x)>0\right.$ for $\left.x \in I\right)$.

Proof. Let $I=\left[x_{0}, \infty\right)$ (the case of $I=\left(-\infty, x_{0}\right]$ can be treated quite similarly). From (21) we have

$$
\left(u u^{\prime}\right)^{\prime}=u^{\prime 2}+u u^{\prime \prime}=u^{\prime 2}+q u^{2} \geqq 0,
$$

and hence $u(x) u^{\prime}(x)$ increases for $x \geqq x_{0}$. Suppose that $u(\bar{x})=0$ for some $\bar{x} \geqq x_{0}$. If $u^{\prime}(\bar{x})=0, u \equiv 0$ by the uniqueness theorem. Thus by the assumption $u^{\prime}(\bar{x}) \neq 0$. If $u^{\prime}(\bar{x})>0$, then $u(x)>0$ for $x$ near $\bar{x}$ and larger than $\bar{x}$. This implies $u(x) u^{\prime}(x)$ $>0$ for $x>\bar{x}$ and hence $u^{\prime}(x)$ increases for $x>\bar{x}$ by $(21)$. Thus $u^{\prime}(x) \geqq u^{\prime}(\bar{x}) \equiv c>0$ and $u(x) \geqq c(x-\bar{x})$ for $x \geqq \bar{x}$. But this contradicts the assumption that $u \in L^{2}(I)$. A similar argument holds in the case where $u^{\prime}(\bar{x})<0$. Thus we obtain $u(x) \neq 0$ for $x \geqq x_{0}$. Consider the case where $u(x)>0$ for $x \in I$ (the case where $u(x)<0$ for $x \in I$ can be treated similarly). Suppose that $u^{\prime}(\bar{x}) \geqq 0$ for some $\bar{x} \in I$. Then $u(x) u^{\prime}(x) \geqq 0$ for $x \geqq \bar{x}$ by (22), and hence $u^{\prime}(x) \geqq 0$ for $x \geqq \bar{x}$. But this implies $u(x) \geqq u(\bar{x})>0$ for $x \geqq \bar{x}$, which contradicts the assumption $u \in L^{2}(I)$. Thus $u^{\prime}(x)$ has to be negative for all $x \in I$, and hence $u(x) u^{\prime}(x)<0$ for $x \in I$.

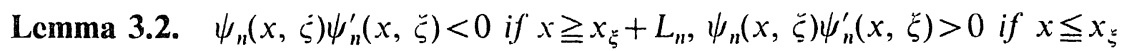
$-L_{n}$, where $x_{\xi}=b^{-1}(\xi)$ and $L_{n}=\sqrt{(2 n-1) M_{+}} / M_{-}$.

Proof. By (12), (19) and (ii) of Lemma 2.3, we have

$$
Q_{n, \xi}(x) \geqq M_{-}^{2}\left(x-x_{\xi}\right)^{2}-(2 n-1) M_{+} \geqq 0
$$

if $\left|x-x_{\xi}\right| \geqq L_{n}$. Therefore, by (20) and Proposition 3.1, we have the assertion of the lemma since $\psi_{n} \in L^{2}\left(\boldsymbol{R}_{x}\right)$.

Lemma 3.3. $\psi_{n}(x, \breve{\zeta}) \rightarrow 0, \psi_{n}^{\prime}(x, \breve{\zeta}) \rightarrow 0 \quad$ as $\quad x \rightarrow \pm \infty$. 
Proof. By Lemma 3.2, $\psi_{n}(x, \xi) \neq 0$ for $x \geqq x_{\xi}+L_{n}$. Suppose that $\psi_{n}(x, \xi)$ $>0$ for $x \geqq x_{\xi}+L_{n}$. Then $\psi_{n}^{\prime}(x, \check{\zeta})<0$ by Lemma 3.2. This implies that $\lim _{x \rightarrow \infty}$ $\psi_{n}(x, \ddot{\zeta})$ exists and $\geqq 0$. Since $\psi_{n} \in L^{2}\left(\mathbb{R}_{x}\right)$, this limit must be 0 . Moreover. by (20) and (23), $\psi_{n}^{\prime \prime}(x, \xi) \geqq 0$ for $x \geqq x_{\xi}+L_{n}$. Thus $\lim _{x \rightarrow \infty} \psi_{n}^{\prime}(x, \xi)$ exists and $\leqq 0$.

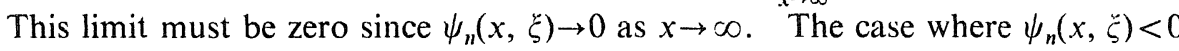
can be treated similarly. A similar argument shows that the limits of $\psi_{n}(x, \xi)$ and $\psi_{n}^{\prime}(x, \xi)$ as $x \rightarrow-\infty$ exist and must be zero.

Lemma 3.4. Let

$$
l_{n}(x, \xi)=\psi_{n}^{\prime}(x, \xi)^{2}-Q_{n, \xi}(x) \psi_{n}(x, \xi)^{2},
$$

where $Q_{n, \xi}$ is as in (19) and let $x_{\xi}=b^{-1}(\xi)$. Then we have the following:

(i) $l_{n}(x, \breve{\zeta})$ is strictly decreasing for $x>x_{\xi}$ and strictly increasing for $x<x_{\xi}$.

(ii) $l_{n}(x, \xi) \rightarrow 0$ as $x \rightarrow \pm \infty$.

(iii) $l_{n}(x, \breve{\zeta})>0$.

Proof. We have

$$
\begin{aligned}
l_{n}^{\prime}(x, \xi) & =2 \psi_{n}^{\prime}(x, \xi)\left\{\psi_{n}^{\prime \prime}(x, \xi)-Q_{n, \xi}(x) \psi_{n}(x, \xi)\right\}-Q_{n, .}^{\prime}(x) \psi_{n}(x, \xi)^{2} \\
& =-Q_{n, \xi}^{\prime}(x) \psi_{n}(x, \xi)^{2} \\
& =-2 B(x)(b(x)-\xi) \psi_{n}(x, \xi)^{2}
\end{aligned}
$$

by (20), (19) and (11). Hence, by (11), (B) and the fact that, for each $\xi$, $\left\{x \mid \psi_{n}(x, \breve{\zeta})=0\right\}$ cannot have an accumulation point, we have (i).

By Lemma 3.2, $\psi_{n}(x, \xi)>0$ for all $x \geqq x_{\xi}+L_{n}$, or $\psi_{n}(x, \breve{\zeta})<0$ for all $x \geqq x_{\xi}+L_{n}$. Consequently, by (20) and (23), we have $\psi_{n}^{\prime \prime}(x, \xi) \geqq 0$ for all $x \geqq x_{\xi}$ $+L_{n}$, or $\psi_{n}^{\prime \prime}(x, \xi) \leqq 0$ for all $x \geqq x_{\xi}+L_{n}$. Thus $\psi_{n}^{\prime}(x, \xi)$ is increasing or decreasing for $x \geqq x_{\xi}+L_{n}$ and tends to 0 as $x \rightarrow \infty$ by Lemma 3.3, from which follows that $\psi_{n}^{\prime \prime}(x, \xi) \rightarrow 0$ as $x \rightarrow \infty$. Hence, by (24), (20) and Lemma 3.3, $l_{n}(x, \xi)=\psi_{n}^{\prime}(x, \xi)^{2}$ $-\psi_{n}^{\prime \prime}(x, \xi) \psi_{n}(x, \xi) \rightarrow 0$ as $x \rightarrow \infty$. Similarly, we have $l_{n}(x, \xi) \rightarrow 0$ as $x \rightarrow-\infty$. Thus, we have obtained (ii). (i) and (ii) imply (iii).

Lemma 3.5. $\left|\psi_{n}(x, \xi)\right| \leqq \Phi_{n}\left(x-x_{\xi}\right)$ where $x_{\xi}=b^{-1}(\xi)$ and

$$
\Phi_{n}(x)=\left\{\begin{array}{l}
\sqrt{2} \lambda_{n}^{+1 / 4} \quad \text { if }|x| \leqq L_{n} \\
\sqrt{2} \lambda_{n}^{+1 / 4} \exp \left\{-\frac{M_{-}}{2}\left(|x|-L_{n}\right)^{2}\right\} \quad \text { if }|x| \geqq L_{n}
\end{array}\right.
$$

with $\lambda_{n}^{+}=(2 n-1) M_{+}, L_{n}=\sqrt{\lambda_{n}^{+}} / M_{-}$.

Proof. First, we have 


$$
\left|\psi_{n}(x, \xi)\right| \leqq \sqrt{2} \lambda_{n}^{+1 / 4} \quad \text { for all } \quad x .
$$

In fact, we have by multiplying (10) by $\psi_{n}(x, \xi)$ and integrating by parts,

$$
\int_{-\infty}^{\infty} \psi_{n}^{\prime}(x, \xi)^{2} d x+\int_{-\infty}^{\infty}(b(x)-\xi)^{2} \psi_{n}(x, \xi)^{2} d x=\lambda_{n}(\xi) \int_{-\infty}^{\infty} \psi_{n}(x, \xi)^{2} d x \leqq \lambda_{n}^{+}
$$

where we have used Lemma 3.3, $\left\|\psi_{n}(\cdot, \xi)\right\|_{L^{2}}=1$ and (ii) of Lemma 2.3. Therefore, we obtain (26) since

$$
\psi_{n}(x, \xi)^{2}=2 \int_{-\infty}^{x} \psi_{n}^{\prime}(x, \xi) \psi_{n}(x, \xi) d x \leqq 2\left(\int_{-\infty}^{x} \psi_{n}^{\prime 2} d x\right)^{1 / 2}\left(\int_{-\infty}^{x} \psi_{n}^{2} d x\right)^{1 / 2} .
$$

Next, by (iii) of Lemma 3.4, we have $\psi_{n}^{\prime}(x, \xi)^{2}>Q_{n, \xi}(x) \psi_{n}(x, \xi)^{2}$. Hence, we have

$$
\begin{array}{cc}
\frac{\psi_{n}^{\prime}(x, \xi)}{\psi_{n}(x, \xi)}<-\sqrt{Q_{n, \xi}(x)} & \text { for } \quad x \geqq x_{\xi}+L_{n} \\
\left(\frac{\psi_{n}^{\prime}(x, \xi)}{\psi_{n}(x, \xi)}>\sqrt{Q_{n, \xi}(x)}\right. & \text { for } \left.\quad x \leqq x_{\xi}-L_{n}\right)
\end{array}
$$

by Lemma 3.2 and (23). Therefore, we obtain

$$
\left|\psi_{n}(x, \xi)\right| \leqq\left|\psi_{n}(\bar{x}, \xi)\right| \exp \left\{-\left|\int_{\bar{x}}^{x} \sqrt{Q_{n, \xi}(t)} d t\right|\right\}
$$

if $x \geqq \bar{x} \geqq x_{\xi}+L_{n}$ (or if $x \leqq \bar{x} \leqq x_{\xi}-L_{n}$ ). Since we have by (23)

$$
Q_{n, \xi}(x) \geqq M_{-}^{2}\left(\left|x-x_{\xi}\right|^{2}-L_{n}^{2}\right) \geqq M_{-}^{2}\left(\left|x-x_{\xi}\right|-L_{n}\right)^{2}
$$

if $\left|x-x_{\xi}\right| \geqq L_{n}$, it follows from (26) and (27) that

$$
\left|\psi_{n}(x, \xi)\right| \leqq \sqrt{2} \lambda_{n}^{+1 / 4} \exp \left\{-\frac{M_{-}}{2}\left(\left|x-x_{\xi}\right|-L_{n}\right)^{2}\right\}
$$

if $\left|x-x_{\xi}\right| \geqq L_{n}$. This completes the proof of the lemma.

Lemma 3.6. $\frac{d}{d \xi} \lambda_{n}(\xi)=\lim _{\substack{A \rightarrow \infty \\ A \rightarrow-\infty}} \int_{A^{\prime}}^{A} \frac{B^{\prime}(x)}{B(x)^{2}} l_{n}(x, \xi) d x$ where $l_{n}(x, \xi)$ is as in Lemma 3.4 .

Proof. Since $\frac{d}{d \xi} \lambda_{n}(\xi)=\left(\frac{d H(\xi)}{d \xi} \psi_{n}(\cdot, \xi), \psi_{n}(\cdot, \xi)\right)$, we have

$$
\begin{aligned}
\frac{d}{d \xi} \lambda_{n}(\xi) & =2 \int_{-\infty}^{\infty}(\xi-b(x)) \psi_{n}(x, \xi)^{2} d x \\
& =2 \int_{-\infty}^{\infty}-\frac{(b(x)-\xi) B(x) \psi_{n}(x, \xi)^{2}}{B(x)} d x \\
& =\lim _{\substack{A \rightarrow \infty \\
A^{A} \rightarrow-\infty}} \int_{A^{\prime}}^{A} \frac{1}{B(x)} l_{n}^{\prime}(x, \xi) d x,
\end{aligned}
$$


where we used (25). Hence we obtain the desired equality by integration by parts with the aid of Lemma 3.4 (ii).

\section{§4. Proof of the Theorem}

We start the proof of the theorem by the following two lemmas, which assert that the asymptotic behavior of $\lambda_{n}(\xi)$ as $\xi \rightarrow \pm \infty$ is determined by that of $B(x)$ as $x \rightarrow \pm \infty$ :

Lemma 4.1. Suppose that $B_{1}(x)$ and $B_{2}(x)$ satisfy the assumption (B). Let $\widetilde{H}_{j}(\xi)$ be the operator $\widetilde{H}(\xi)$ in Lemma 2.3 with $B$ replaced $b{ }^{\prime} B_{j}$ and let $\lambda_{n}^{(j)}(\xi)$ be the $n-1$ h eigenvalue of $\tilde{H}_{j}(\xi)(j=1,2)$. Assume that $B_{1}(t)-B_{2}(t) \rightarrow 0$ as $t \rightarrow \infty \quad(t \rightarrow-\infty)$. Then, for each $n, \lambda_{n}^{(1)}\left(b_{1}(s)\right)-\lambda_{n}^{(2)}\left(b_{2}(s)\right) \rightarrow 0$ as $s \rightarrow \infty$ $\left(s \rightarrow-\infty\right.$, respectively), where $b_{j}(x)=\int_{0}^{x} B_{j}(t) d t(j=1,2)$.

Proof. We show that the Lemma holds under the assumption $B_{1}(t)-B_{2}(t)$ $\rightarrow 0$ as $t \rightarrow \infty$. The case where $B_{1}(t)-B_{2}(t) \rightarrow 0$ as $t \rightarrow-\infty$ can be treated similarly.

Let $n$ be fixed and let $j, k \leqq n$. Let

$$
\alpha_{j k}(s) \equiv\left(\widetilde{H}_{2}\left(b_{2}(s)\right) \psi_{j}^{(1)}\left(\cdot, b_{1}(s)\right), \psi_{k}^{(1)}\left(\cdot, b_{1}(s)\right)\right)
$$

where $\psi_{j}^{(1)}(x, \xi)$ is the eigenfunction of $\tilde{H}_{1}(\xi)$ with the eigenvalue $\lambda_{j}^{(1)}(\xi)$ as in Lemma 2.3. Then, since

$$
\left(\tilde{H}_{1}\left(b_{1}(s)\right) \psi_{j}^{(1)}\left(\cdot, b_{1}(s)\right), \psi_{k}^{(1)}\left(\cdot, b_{1}(s)\right)\right)=\lambda_{j}^{(1)}\left(b_{1}(s)\right) \delta_{j k}
$$

where $\delta_{j k}=1(j=k)$ and $0(j \neq k)$, we have

$$
\begin{aligned}
& \left|\alpha_{j k}(s)-\lambda_{j}^{(1)}\left(b_{1}(s)\right) \delta_{j k}\right| \\
& \quad \leqq \int_{-\gamma}^{\infty}\left|\left(b_{2}(x)-b_{2}(s)\right)^{2}-\left(b_{1}(x)-b_{1}(s)\right)^{2}\right|\left|\psi_{j}^{(1)}\left(x, b_{1}(s)\right)\right|\left|\psi_{h}^{(1)}\left(x, b_{1}(s)\right)\right| d x .
\end{aligned}
$$

On the other hand, we have by (B) with $B=B_{1}$ or $B_{2}$,

$$
\begin{aligned}
& \left|\left(b_{2}(x)-b_{2}(s)\right)^{2}-\left(b_{1}(x)-b_{1}(s)\right)^{2}\right| \\
& \quad=\left|\left(\int_{s}^{x}\left(B_{1}(t)+B_{2}(t)\right) d t\right)\left(\int_{s}^{x}\left(B_{1}(t)-B_{2}(t)\right) d t\right)\right| \\
& \quad \leqq 2 M_{+}|x-s|\left|\int_{s}^{x}\left(B_{1}(t)-B_{2}(t)\right) d t\right| .
\end{aligned}
$$

Moreover, we have, by Lemma 3.5 and by noting that $\Phi_{1}(x)<\Phi_{2}(x)<\cdots<$ $\Phi_{n}(x)$,

$$
\left|\psi_{j}^{(1)}\left(x, b_{1}(s)\right)\right| \leqq \Phi_{n}(x-s) \quad(j=1,2, \ldots, n) .
$$


Thus, from (29), (30) and (31), we obtain for $j, k \leqq n$

$$
\begin{aligned}
& \left|\alpha_{j h}(s)-\lambda_{j}^{(1)}\left(b_{1}(s)\right) \delta_{j h}\right| \\
& \quad \leqq 2 M_{+} \int_{-\infty}^{\infty}\left\{|x-s|\left|\int_{s}^{x}\left(B_{1}(t)-B_{2}(t)\right) d t\right| \Phi_{n}(x-s)^{2}\right\} d x .
\end{aligned}
$$

Let $\varepsilon>0$. Then there exists a real number $R$ such that $\sup _{R \leqq t}\left|B_{1}(t)-B_{2}(t)\right| \leqq \varepsilon$ by the assumption. Hence, by (32) and (B), we have for $s \geqq R$,

$$
\begin{aligned}
& \left|\alpha_{j k}(s)-\lambda_{j}^{(1)}\left(b_{1}(s)\right) \delta_{j k}\right| \\
& \quad \leqq 4 M_{+}^{2} \int_{-\infty}^{R}|x-s|^{2} \Phi_{n}(x-s)^{2} d x+2 M_{+} \varepsilon \int_{R}^{\infty}|x-s|^{2} \Phi_{n}(x-s)^{2} d x \\
& \quad \leqq 4 M_{+}^{2} \int_{-\infty}^{R-s} x^{2} \Phi_{n}(x)^{2} d x+2 M_{+} \varepsilon \int_{-\infty}^{\infty} x^{2} \Phi_{n}(x)^{2} d x .
\end{aligned}
$$

Hence, by noting that $\Phi_{n}(x)$ depends only on $n, M_{-}$and $M_{+}$, and $x^{2} \Phi_{n}(x)^{2}$ is integrable on $\boldsymbol{R}$, we have by (33)

$$
\left|\alpha_{j k}(s)-\lambda_{j}^{(1)}\left(b_{1}(s)\right) \delta_{j k}\right| \leqq C \varepsilon
$$

for sufficiently large s and for $j, k \leqq n$, where $C$ is a constant dependent only on $n, M_{-}$and $M_{+}$. Let $V_{n}(s)$ be the linear subspace of $L^{2}\left(\boldsymbol{R}_{x}\right)$ spanned by $\left\{\psi_{j}^{(1)}(\cdot\right.$, $\left.\left.b_{1}(s)\right)\right\}_{j=1, \ldots, n}$ and let $R_{n}(s)$ be the orthogonal projection onto $V_{n}(s)$. Then we have by (28)

$$
\left\{\begin{array}{l}
\left(\alpha_{j k}(s)\right) \text { is the Hermitian symmetric matrix of } \\
\left.R_{n}(s) \widetilde{H}_{2}\left(b_{2}(s)\right) R_{n}(s)\right|_{V_{n}(s)} \text { with respect to the basis } \\
\left\{\psi_{j}^{(1)}\left(\cdot, b_{1}(s)\right)\right\}_{j=1, \ldots, n} .
\end{array}\right.
$$

Let $\mu_{1}(s) \leqq \cdots \leqq \mu_{n}(s)$ be the eigenvalues of $\left(\alpha_{j k}(s)\right)$. Then we have by (35) and by applying the min-max principle to the operator $\widetilde{H}_{2}\left(b_{2}(s)\right)$ (see [9], p. 270, Lemma)

$$
\lambda_{j}^{(2)}\left(b_{2}(s)\right) \leqq \mu_{j}(s) \quad(j=1, \ldots, n) .
$$

On the other hand we have by (34)

$$
\left|\mu_{j}(s)-\lambda_{j}^{(1)}\left(b_{1}(s)\right)\right| \leqq C^{\prime} \varepsilon \quad\left(C^{\prime}=n C, j=1, \ldots, n\right)
$$

for sufficiently large $s$. Thus for any $\varepsilon>0$, the following inequality holds for sufficiently large $s$ by (36) and (37):

$$
\lambda_{j}^{(2)}\left(b_{2}(s)\right) \leqq \lambda_{j}^{(1)}\left(b_{1}(s)\right)+C^{\prime} \varepsilon \quad(j=1, \ldots, n),
$$

where $C^{\prime}$ is a constant dependent only on $n, M_{-}$and $M_{+}$. By interchanging 
the subscripts 1 and 2 in the above argument, we have

$$
\lambda_{j}^{(1)}\left(b_{1}(s)\right) \leqq \lambda_{j}^{(2)}\left(b_{2}(s)\right)+C^{\prime} \varepsilon \quad(j=1, \ldots, n)
$$

for sufficiently large s. By (38) and (39), for each $n$,

$$
\lambda_{n}^{(1)}\left(b_{1}(s)\right)-\lambda_{n}^{(2)}\left(b_{2}(s)\right) \longrightarrow 0 \text { as } s \longrightarrow \infty .
$$

This proves the lemma.

Lemma 4.2. $\quad(2 n-1) \liminf _{x \rightarrow \pm \infty} B(x) \leqq \liminf _{\xi \rightarrow \pm \infty} \lambda_{n}(\xi)$ and $\limsup _{\xi \rightarrow \pm \infty} \lambda_{n}(\xi) \leqq(2 n-1)$ $\limsup _{x \rightarrow \pm \infty} B(x)$.

Proof. Let $B_{-} \equiv \liminf _{x \rightarrow \infty} B(x)$ and let $\varepsilon>0$. Then, there exists a real number $R$ such that $B(x) \geqq B_{-}-\varepsilon$ for $x \geqq R$ and hence there exists a $C^{\infty}$ function $B_{1}(x)$ satisfying the assumption (B) and such that $B_{1}(x)=B(x)$ for $x \geqq R$ and $B_{1}(x)$ $\geqq B_{-}-2 \varepsilon$ for all $x$. Then, by applying Lemma 4.1 to this $B_{1}(x)$ and $B_{2}(x) \equiv$ $B(x)$, we have that, for each $n$,

$$
\lambda_{n}^{(1)}\left(b_{1}(s)\right)-\lambda_{n}^{(2)}\left(b_{2}(s)\right) \longrightarrow 0 \text { as } s \longrightarrow \infty .
$$

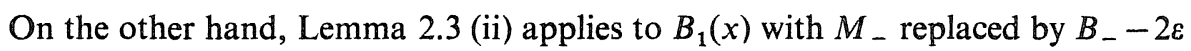
since $B_{1}(x) \geqq B_{-}-2 \varepsilon$ for all $x$. Thus we have

$$
\lambda_{n}^{(1)}(\xi) \geqq(2 n-1)\left(B_{-}-2 \varepsilon\right)
$$

for all $\xi \in \mathbb{R}$ and for $n=1,2, \ldots$. It follows from (40) and (41) that

$$
\liminf _{s \rightarrow \infty} \lambda_{n}^{(2)}\left(b_{2}(s)\right) \geqq(2 n-1)\left(B_{-}-2 \varepsilon\right) .
$$

Thus, since $\varepsilon$ was arbitrary and since $\lambda_{n}^{(2)}(\xi)=\lambda_{n}(\xi)$ and $b_{2}(s) \rightarrow \infty$ as $s \rightarrow \infty$, we have

$$
\liminf _{\xi \rightarrow \infty} \lambda_{n}(\xi) \geqq(2 n-1) B_{-}=(2 n-1) \liminf _{x \rightarrow \infty} B(x),
$$

which proves the first inequality where the double sign is + . We can obtain the remaining inequalities by an argument similar to the above.

Proof of the Theorem. Note that, by Lemma 2.6, it suffices to show that, for each $n, \lambda_{n}(\xi)$ is not constant.

In the case where the assumption (B1) holds, $\lambda_{n}(\xi)$ is not constant since $\limsup _{\xi \rightarrow \mp \infty} \lambda_{n}(\xi)<\liminf _{\xi \rightarrow \pm \infty} \lambda_{n}(\xi)$ by Lemma 4.2 and (B1).

Let us consider the case where (B2) holds. Let $B^{\prime}(x) \leqq 0$ for $x \leqq \bar{x}, B^{\prime}(x) \geqq 0$ for $x \geqq \bar{x}$, and let $R$ be a constant such that $B(x)=B_{0}$ for $|x| \geqq R$. Then we have 


$$
\int_{-\infty}^{\infty} \frac{B^{\prime}(x)}{B(x)^{2}} d x=\int_{-R}^{R} \frac{B^{\prime}(x)}{B(x)^{2}} d x=\frac{1}{B(-R)}-\frac{1}{B(R)}=0
$$

Hence we obtain by Lemma 3.6

$$
\frac{d}{d \xi} \lambda_{n}(\xi)=\int_{-R}^{R} \frac{B^{\prime}(x)}{B(x)^{2}}\left(l_{n}(x, \xi)-l_{n}(\bar{x}, \xi)\right) d x .
$$

On the other hand, if we let $\xi$ be so large that $x_{\xi}>R$ (note that, since $b(x)=$ $\int_{0}^{x} B(t) d t \rightarrow \infty$ as $x \rightarrow \infty, x_{\xi}=b^{-1}(\xi) \rightarrow \infty$ as $\left.\xi \rightarrow \infty\right)$, then $l_{n}(x, \xi)$ is strictly increasing in $[-R, R]$ by (i) of Lemma 3.4. Thus we have that $l_{n}(x, \xi)-l_{n}(\bar{x}, \xi)<0$ for $x<\bar{x}, l_{n}(x, \xi)-l_{n}(\bar{x}, \xi)>0$ for $x>\bar{x}$ and hence $B^{\prime}(x)\left(l_{n}(x, \xi)-l_{n}(\bar{x}, \xi)\right)$ is non-negative and does not vanish identically for $x \in[-R, R]$ by the assumption (B2). Consequently, we have by (42) that $\frac{d}{d \xi} \lambda_{n}(\xi)>0$. Thus $\lambda_{n}(\xi)$ is nonconstant for all $n$. The case where $B^{\prime}(x) \geqq 0$ for $x<\bar{x}, B^{\prime}(x) \leqq 0$ for $x>\bar{x}$ can be treated similarly. This completes the proof of the theorem.

Finally, we remark that, by examining the above argument, one is able to determine the spectrum of $H$ in the case where $B(x)$ is assumed to be monotone in addition to (B). In fact, if we let, e.g., $B(x)$ be increasing, then Lemma 2.3 (ii) holds with $M_{ \pm}$replaced by $B_{ \pm} \equiv \lim _{x \rightarrow \pm \infty} B(x)$ and hence $\sup _{\xi} \lambda_{n}(\xi)=(2 n-1) B_{+}$,

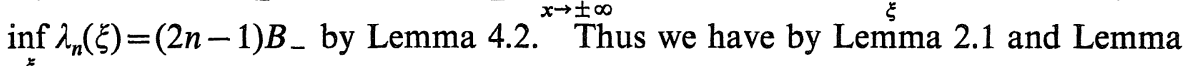
2.5

$$
\sigma(H)=\bigcup_{n=1}^{\infty}\left[(2 n-1) B_{-},(2 n-1) B_{+}\right]
$$

\section{References}

[1] Avron, J., Herbst, I. and Simon, B., Schrödinger operators with magnetic fields I. General interactions, Duke Math. J., 45 (1978), 847-883.

[2] Ikebe, T. and Saito, Y., Limiting absorption method and absolute continuity for the Schrödinger operators, J. Math. Kyoto Univ., 7 (1972), 513-542.

[3] Iwatsuka, A., The essential spectrum of two-dimensional Schrödinger operators with perturbed constant magnetic fields, J. Math. Kyoto Univ., 23 (1983), 475-480.

[4] Kato, T., Perturbation Theory for Linear Operators, Springer, Berlin-Heidelberg-New York, 1976.

[ 5 ] Leinfelder, H., Gauge invariance of Schrödinger operators and related spectral properties, J. Op. Theory, 9 (1983), 163-179.

[6] Leinfelder, H. and Simader, C. G., Schrödinger operators with singular magnetic potentials, Math. Z., 176 (1981), 1-19.

[7] Miller, K. and Simon, B., Quantum magnetic Hamiltonians with remarkable spectral properties, Phys. Rev. Lett., 44 (1980), 1706-1707. 
[8] Reed, M. and Simon, B., Methods of Modern Mathematical Physics, Vol. II, Academic Press, New York, 1975.

[9] —-, Methods of Modern Mathematical Physics, Vol. IV, Academic Press, New York, 1978.

[10] Titchmarsh, E. C., Eigenfunction Expansions Associated with Second-Order Differential Equations, Part I (Second Edition), Oxford Univ. Press, London-New York, 1962. 
